\title{
Physical State of Outer Atmosphere and the Origin of Radiation Belts*
}

\author{
By Tatsuzo OBAYASHI \\ Radio Research Laboratories, Kokubunji, Tokyo \\ (Read November 27, 1959 ; Received January 22, 1960)
}

\begin{abstract}
A possible mechanism trapping high energy particles in the radiation belts surrounding the earth is proposed, taking into account the existing hydromagnetic waves in the outer atmosphere. It is shown that there are two regions where the amplitude of hydromagnetic waves and the compressibility of gas bearing magnetic field are large. Since the acceleration mechanism is operative in such regions, particles inside the regions may be raised in their energies, and consequently they produce local inhomogeneities of hot plasmas. These hot plasmas interact with the geomagnetic field and may form a certain kind of magnetic bottles, in which the high energy particles are likely trapped. The inner activated region is expected at the height $1,000 \sim 3,000 \mathrm{~km}$ and the outer region is of the order of $20,000 \mathrm{~km}$. Although the origin of high energy particles is possibly of injected solar particles, their concentration at particular regions may be controlled considerably by the hydromagnetic nature in the outer atmosphere.
\end{abstract}

\section{Introduction}

During the past few years, considerable advance have been achieved in the knowledge of the upper atmosphere beyond the ionosphere. New evidence indicating the existence of regions trapping high energy particles has been discovered by Van Allen et al [1958], which are now called the radiation belts. These are two distinct zones of intense flux of energetic particles, the inner zone is situated at a few thousand kilometers above the earth's surface, while the outer zone is at the distance of $3 \sim 4$ earth radii.

It is the purpose of this paper to suggest a possible mechanism for trapping such hhig energy particles in the outer atmosphere, though several other mechanisms have already been proposed by different authors. The hydromagnetic waves and the interaction of the geomagnetic field and the ionized outer atmospheric gas are taken into account, and it is shown that a certain type of magnetic bottles may exist as the particle reservoir in the outer atmosphere.

\section{Hydromagnetic Waves in the Outer Atmosphere}

It is known that in an ionized gas in a magnetic field hydromagnetic waves

\footnotetext{
* Read at the First International Space Science Symposium, Nice, Jan. 1960.
} 
appear due to the interaction of fluid motions with the magnetic field. Waves, known as Alfvén waves, are propagated along the magnetic lines of force with a velocity.

$$
V_{A}=\frac{H}{\sqrt{4 \pi \rho}}
$$

whepe $H$ is the magnetic field intensity and $\rho$ is the ionic density in the medium. Besides this Alfvén wave, hydromagnetic waves have two other modes; the modified Alfven waves and the retarded sound wave. In particular, for the propagation of waves parpendicular to the magnetic lines of force, the velocity of the Alfven wave and the retarded sound wave is zero, but the modified Alfvén wave has a velocity

$$
V=\sqrt{\frac{H^{2}}{4 \pi \rho}+V_{s}^{2}}
$$

where $V_{s}=\sqrt{2 k T / m}$, sound wave velocity. This wave, in which the particle velocity is parallel to the direction of propagation, both being perpendicular to $H$, is a longitudinal hydromagnetic wave.

There is a possibility that such waves may be generated in the earth's outer atmosphere due to excitation by solar corpuscular streams, and produce hydromagnetic oscillations. It has been shown elsewhere [Obayashi, 1958] that there exist two kinds of mode of oscillations in the outer atmosphere. The one is caused by a transversal hydromagnetic wave (Alfvén wave) propagating along the geomagnetic line of force, whose oscillation is perpendicular both to the direction of wave propagation and the magnetic field, and each surface of revolution of a geomagnetic line of force oscillates independently. The other is the longitudinal oscillation, the disturbances propagate across the geomagnetic lines of force as the longitudinal wave mode, and the oscillation is set up between the top of the ionosphere and the outer boundary of the earth's atmosphere where solar plasmas are invading.

There is considerable evidence to believe that geomagnetic pulsations observed at high latitudes are mainly the transversal type, while at low latitude the oscillation of longitudinal waves prevails. The longitudinal hydromagnetic waves may have an important effect on the formation of radiation belts, because of their heating or accelerating actions of atmospheric gas. An idea, that hydromagnetic waves are responsible for producing high intensity particle radiation, has been first suggested by Dessler [1958], and he has shown that existing large hydromagnetic waves with sharp crests above $1000 \mathrm{~km}$ are favourable for the Fermi type particle acceleration process. However, as will be described, it is shown that this mechanism producing high energy particles is not sufficient enough to account for the observed energy spectrum of radiation belts. Therefore, some re-consideration of this theory will be made in the following section.

\section{Origin of Radiation Belts}

In an agitated plasma bearing magnetic field, high-speed charged particles gain their energies by colliding with moving clumps of magnetic field or hydromagnetic 
waves, and thus undergo Fermi acceleration [Fermi, 1954]. According to Parker [1958], the effectiveness of the Fermi acceleration depends on two factors, one is the amplitude of hydromagnetic waves and the other is the compressibility of the plasma. As has been pointed out [Obayashi, 1958], there is an evidence that longitudinal hydromagnetic oscillations exist in the outer atmosphere which are excited by solar corpuscular streams. The mode of such oscillations depends on the ionic density and the geomagnetic field distribution in the outer atmosphere. Using the current model of the outer atmosphere shown in Fig. 1 (due to rockets and whistler observations), the velocity of a long-

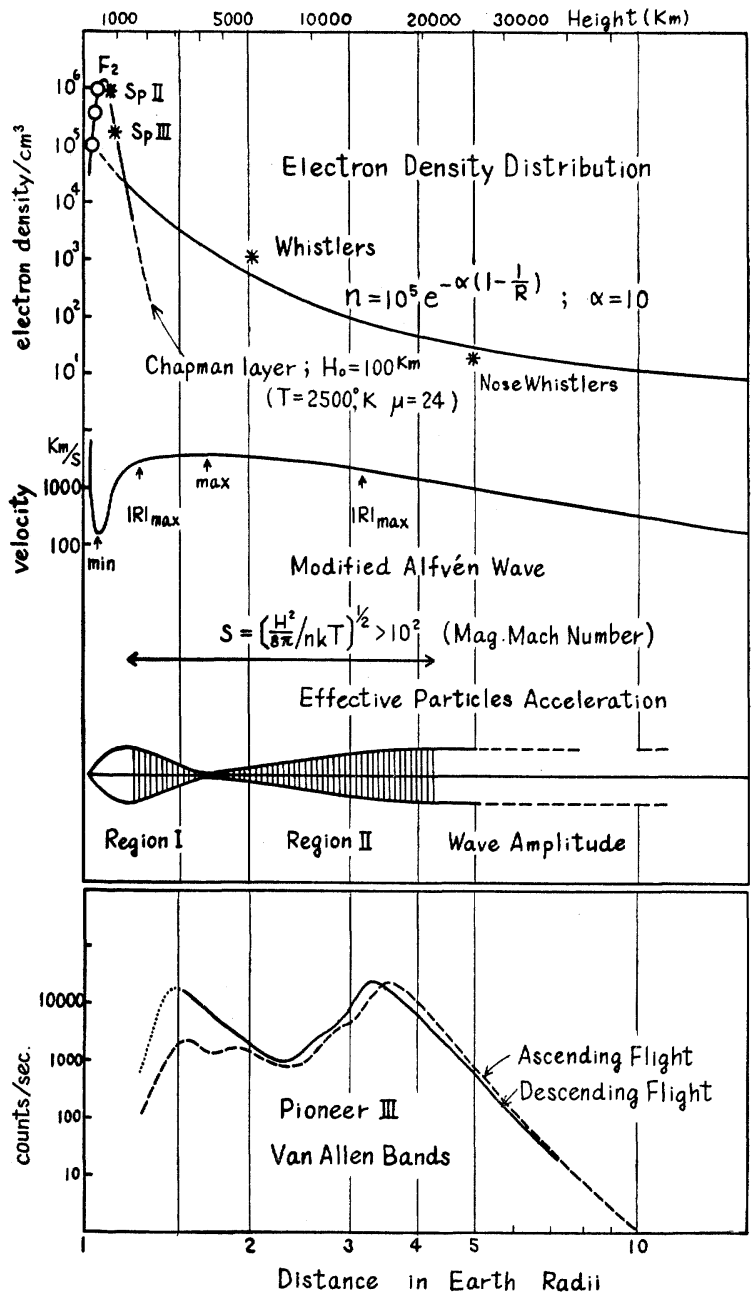

Fig. 1 Hydromagnetic oscillations in the outer atmosphere and the position of the radiation belts itudinal hydromagnetic wave is computed using eq. (2). It is clear that there are two regions where the wave velocity decrease with height. As has been mentioned by Dessler, in these regions downward travelling hydromagnetic waves will be reflected back. Hence, it is likely that the hydromagnetic oscillation may attain a large amplitude at the fringe of the ionosphere near the height of $1,000 \mathrm{~km}$ and above $10,000 \mathrm{~km}$ while it has a kind of nodal point in the ionosphere and at $5,000 \mathrm{~km}$ above the earth's surface.

On the other hand, the Fermi process can be operative only as long as the hydromagnetic waves can maintain sharp fronts or crests. This situation is possible only when the compressibility of the gas bearing magnetic inhomogeneities[ $\Delta H^{2}$ $/ 8 \pi n k T]$ is large. In Fig. 1 , the region of the magnetic Mach number larger than $10^{2}$ is indicated by the line. Thus, it can be understood that there are two regions where the situation is favorable

for atmospheric gas particles to be accelerated by hydromagnetic waves. As shown by hatching in Fig. 1, the region I exists at the height between $1,000 \sim 3,000 \mathrm{~km}$, and the region II is of the height between $10,000 \sim 20,000 \mathrm{~km}$. It is worthwhile to note that they are situated at the place where the radiation belts have been found.

The energy spectrum of accelerated particles by the Fermi mechanism (non rela- 
tivistic) is given [Parker 1957],

$$
j(W)=\frac{1}{2 N_{0} m V^{2}} \exp \left[-\frac{W-W_{0}}{2 N_{0} m V^{2}}\right]
$$

where $W_{0}$ is the energy of thermal ions in the outer atmosphere. $N_{0}$ is number of collisions of a particle with hydromagnetic wave of the wave length $\lambda$ before escaping from the agitated region of dimension $L$. If particles undergo random walk in the region, $N_{0}$ is given by $(L / \lambda)^{2}$. Assuming that $V=1,000 \mathrm{~km} / \mathrm{s}, \lambda=5,000 \mathrm{~km}$ and $L=10,000$ $\mathrm{km}$ it can be shown that the energy of protons attained by this process is

$$
W^{*} \sim 2 N_{0} m V^{2} \simeq 100 \mathrm{KeV}
$$

Therefore, the Fermi acceleration is not sufficient to produce the observed energy, although if an equipartition of energy between the electrons and the accelerated protons is achieved, the existing electrons in the outer radiation zone may be explainable by this mechanism [Singer, 1959]

However, since the regions activated by hydromagnetic waves are situated at the place where the radiation belts have been found (see bottom of Fig. 1), the following discussion may be appropriate: Owing to the continual agitation by hydromagnetic waves, part of the particles inside the activated regions may be raised their energies. Those high energy particles will suffer collisions and change their kinetic energy to heat. The heating may also be due to the violent beating of neutral gas by hydromagnetic waves. Consequently, the continual heating of the outer atomsphere by such processes may generate local inhomogeneities of hot plasmas. It is suggested that those hot plasmas expand outwards interacting with the existing geomagnetic field. The geomagnetic configuration may be altered to some extent like a magnetic bottle, shown in Fig. 2. Two regions of such magnetic bottles are expected, where high

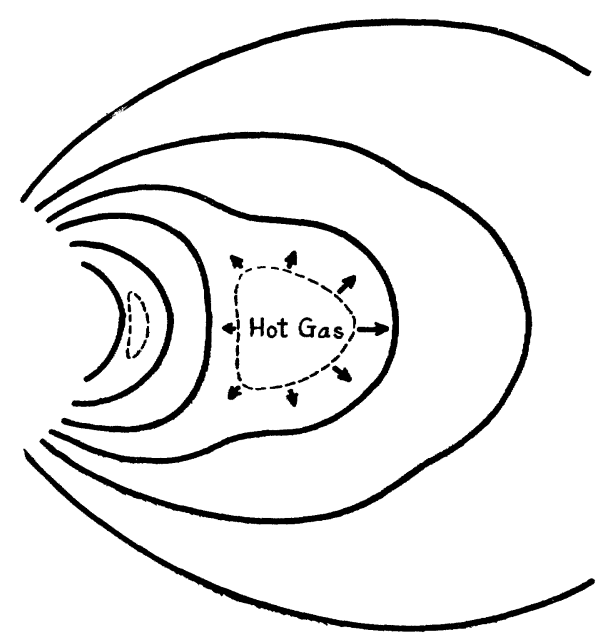

Fig. 2 Distortion of the outer geomagnetic field due to agitated hot plasmas energy particle are trapped more firmly. This may be consistent with the result of the measured outer geomagnetic field by the "Cosmic Rocket" [Dolginov and Pushkov, 1959], which shows a slight drop of scalar field intensity below the theoretically expected one in the radial distance range $15,000 \sim 22,000$ $\mathrm{km}$.

The origin of high energy particles is still uncertain. However, observation by Pioneer IV, which was launched after a geomagnetic storm, indicates an increase of particle flux even at the inner radiation belt [Van Allen and Frank, 1959]. The height of the inner belt decreases considerably during magnetic storms [Yoshida, 1959]. These results suggest that the injection of solar particles is likely the origin of radiation 
belts, and also that the activation by hydromagnetic waves may take an important role to maintain and control the radiation belts.

\section{Acknowledgement}

The author wishes to express his thanks to Prof. K. Maeda, Kyoto University, for his encouragement throughout the study and also to Prof. S. Hayakawa, Nagoya University, for his kind suggestions on this problem.

\section{References}

Dessler A. J. (1958) Large amplitude hydromagnetic waves above the ionosphere, J. Geophys. Res. 63, 507.

Dolginov S. and Pushkov N. (1959) International Conference of Cosmic Ray (IUPAP), July, Moscow.

Fermi E. (1954) Galactic fields and the origin of cosmic ray, Astrophys. J. 119, 1.

Obayashi T. (1958) Geomagnetic storms and the earth's outer atmosphere, Rep. Ionos. Res. Japan, 12, 301.

Parker E. N. (1957) Acceleration of cosmic rays in solar flares, Phys. Rev. 107, 830.

Parker E. N. (1958) Origin and dynamics of cosmic rays, Phys. Rev. 109, 1328.

Singer S. F. (1959) Trapped radiation in the earth's magnetic field, Univ. Maryland, Phys. Dept. Tech. Rep. No. 153.

Van Allen J.A., Mcllwain C.E. and Ludwig G. L. (1958) Radiation observation with satellite, Trans. Amer. Geophys. Union, 39, 767.

Van Allen J. A. and Frank L.A. (1959) Radiation around the earth to a radial distance of 107,400 km, Nature, 183, 430.

Van Allen J.A. and Frank L.A. (1959) Radiation measurements to $658,300 \mathrm{~km}$ with Pioneer IV, Nature 184, 219.

Yoshida S. (1959) Private communication. 